\title{
EVALUATING ANALOG VS HUMAN INSULIN EFFICACY IN REAL LIFE. OBSERVATIONAL STUDY IN TYPE 2 ALBANIAN DIABETIC PATIENTS, PREVIOUSLY INSULIN TREATED
}

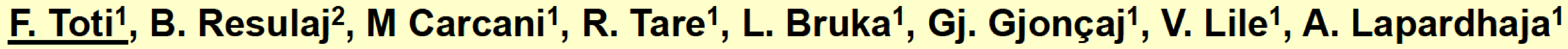

1 Endocrinology \& Metabolic diseases, UHCenter "Mother Theresa", Tirana, Albania.

2 Faculty of Medicine, Medical University, Tirana, Albania

\section{OBJECTIVES}

Insulin therapy is an important part of diabetes treatment.

In Albania, specialists still have to demonstrate at the institutions the treatment's efficacy and cost-effectiveness for new insulin analogs.

The aim of our study is to evaluate the efficacy of analogs vs human insulins and differences between various analog insulin, in type 2 diabetes patients, previously treated with human insulin.

\section{METHODS}

- This study is realized in real life patients

- We retrospectively included 384 patients, previously treated $\geq 24$ months with human insulins, switched to an analog insulin for $\geq 12$ months.

- Treatment efficacy was evaluated by HbA1c levels, weight difference and changes in total daily dose (TDD) analog vs human.

\section{RESULTS}

- $\quad 384 p t s ~(48.17 \%)$ males. Mean age 62.19 (SD 10.12) yrs, mean diabetes duration 10.8 (SD5.35) yrs. Mean duration on analog insulin therapy was 19.1 months.

- GLargine 194 (50.5\%), DEtemir 110 (28.6\%), All Other Analogs (AOA) $80(20.8 \%)$.

- Averall Mean HbA1c was 8.86(SD1.06) vs 7.51(SD1.51) on analogs $p<0.01$. TDD was $54.9 \mathrm{UI}$ (SD20.1) vs 62.56UI (SD27.95) on analogs $p<0.05$, but smaller basal doses 29.28 vs $28.1 \mathrm{Ul}$.

- $18 \%$ of the patients on human insulin has an $\mathrm{HbA} 1 \mathrm{c}<7 \%$, vs $55.1 \%$ on analogs $(p<0.01)$.

- Patients on analogs had a slight weight increase $+3.18 \mathrm{~kg}$ during the study period ( 0.55$)$, but DE/GL 1.48 vs 4.14 $\mathrm{kg}(\mathrm{p}<0.05)$.

\begin{tabular}{|c|c|c|c|c|c|c|c|}
\hline & PATIENTS & $\begin{array}{c}\text { MEAN } \\
\text { DIABETES } \\
\text { DURATION } \\
\text { (yrs) }\end{array}$ & $\begin{array}{c}\text { MEAN TREATMENT } \\
\text { TIME } \\
\text { (months) }\end{array}$ & $\begin{array}{l}\text { CHANGES OF } \\
\text { HbA1C SINCE THE } \\
\text { INITIATION OF } \\
\text { ANALOGS }\end{array}$ &  & $\begin{array}{c}\text { MEAN DAILY } \\
\text { DOSE OF } \\
\text { ANALOGS }\end{array}$ & $\begin{array}{c}\text { WEIGHT } \\
\text { CHANGES }\end{array}$ \\
\hline Ins. GLARGINE & 194 & 12.19 & 19.08 & $\begin{array}{c}7.16 / 8.51 \\
-1.35 \%\end{array}$ & $\begin{array}{c}56.5 \text { vs } \\
18.3 \%\end{array}$ & 30.89 & $+4.14 \mathrm{~kg}$ \\
\hline Ins. DETEMIR & 110 & 10.98 & 19.93 & $\begin{array}{c}6.81 / 8.17 \\
-1.36 \%\end{array}$ & $\begin{array}{c}63.4 \text { vs } \\
20.7 \%\end{array}$ & 27.95 & $+1.48 \mathrm{~kg}$ \\
\hline $\begin{array}{l}\text { ALL OTHER } \\
\text { ANALOGS }\end{array}$ & 80 & 11.72 & 15.22 & $\begin{array}{c}7.16 / 8.57 \\
-1.38 \%\end{array}$ & $\begin{array}{c}45.5 \text { VS } \\
15.2 \%\end{array}$ & 22.56 & $+2.54 \mathrm{~kg}$ \\
\hline TOTAL & 384 & 11.7 & 18.88 & $\begin{array}{c}6.96 / 8.31 \\
-1.35 \%\end{array}$ & $\begin{array}{c}55.1 \text { vs } \\
18.1 \%\end{array}$ & 29.28 & $+3.18 \mathrm{~kg}$ \\
\hline
\end{tabular}

\section{CONCLUSIONS}

- A better metabolic control was noted with analog vs human insulins, with smaller daily doses of basal insulin and minimal weight increase.

- Even in our study Detemir group had a smaller weight gain, making it preferable for obese type 2 diabetics. 